\title{
Clinical assessment, gait analysis and reliability of measurement with the $G$ walk inertial sensor in subjects with ankle fracture 6 months after surgery.
}

\section{Mario Fernández-Gorgojo}

Escuela Universitaria Gimbernat Cantabria: Escoles Universitaries Gimbernat i Tomas Cerda - Campus

Torrelavega

Diana Salas-Gómez ( $\sim$ diana.salas@eug.es)

Escuela Universitaria Gimbernat Cantabria: Escoles Universitaries Gimbernat i Tomas Cerda - Campus

Torrelavega https://orcid.org/0000-0001-6794-7002

\section{Pascual Sánchez-Juan}

Hospital Universitario Marques de Valdecilla

\section{David Barbado}

Universidad Miguel Hernández: Universidad Miguel Hernandez de Elche

\section{Esther Laguna-Bercero}

Hospital Universitario Marques de Valdecilla

Maria Isabel Pérez-Núñez

Hospital Universitario Marques de Valdecilla

\section{Research Article}

Keywords: Ankle fracture, gait analysis, inertial sensor unit, spatial-temporal parameters, functional assessment, reliability

Posted Date: February 2nd, 2022

DOI: https://doi.org/10.21203/rs.3.rs-1288386/v1

License: (c) (i) This work is licensed under a Creative Commons Attribution 4.0 International License.

Read Full License 


\section{Abstract}

Background: Ankle fractures can cause significant functional impairment in the short and long term. In recent years, gait analysis using inertial sensors has gained special relevance as a reliable measurement system.

Objectives: In patients with bimalleolar ankle fracture, to identify the differences between the operated and healthy ankle in the spatial-temporal gait parameters and plantar pressures; likewise, to study their relationship with clinical parameters and functional scales. Finally, to assess the reliability of a single inertial sensor, placed on the lumbar spine, in our study population.

Design: Cross-sectional study.

Methods: Twenty-two subjects with bimalleolar ankle fractures were included in the study. An analysis of the spatial-temporal gait parameters and plantar pressures was carried out using an inertial system (GWALK) and a pressure platform (P-WALK). Sociodemographic and clinical information was previously collected. Differences between the operated and healthy ankle were studied for each variable and their correlation with clinical measures and functional scales; then, a multiple linear regression analysis was performed with the variables that showed a significant correlation. Finally, the intra-session test-retest reliability of the $\mathrm{G}$ Walk was calculated using the intraclass correlation coefficient $\left(\mathrm{ICC}_{2,1}\right)$ and the standard error of measurement (SEM).

Results: Statistically significant differences were found for all spatial-temporal gait parameters and dynamic plantar pressures, except for double support (-1\%; $p=.267)$. Differences were also found for clinical measures, except for ADD strength ( $0.6 \% ; p=.491)$. Effect sizes for gait variables ranged from 0.25 to 1.02 and for clinical variables ranged from 0.15 to 2.30 . The highest degree of correlation of clinical measures and functional scales was obtained with speed, cadence, stride time, and propulsion. Intrasession test-retest reliability was high to excellent $(0.84<I C C>0.98)$. Speed, stride length, and propulsion obtained the lowest SEM scores.

Conclusions: In our study population, the analysis of spatial-temporal gait parameters and dynamic plantar pressures show an evident asymmetry between both limbs; likewise, they correlate moderately with ankle range of motion, hip abductor strength, and the AOFAS scale. The G-WALK inertial sensor can be considered a reliable gait measurement system in this population.

\section{Background}

Ankle fractures represent $10 \%$ of all bone fractures, with bimalleolar or lateral malleolus fractures being the most common according to the selection criteria used in the studies [1,2]. The incidence has been increasing over the last two decades to between 71-187 fractures per 100,000 people depending on age, sex, and geographic region [3]. Surgical treatment of these fractures is necessary when joint congruence 
cannot be restored by conservative treatment, as instability, misalignment, and residual displacements will lead to short- and long-term functional impairment [4-9].

The importance of the severity of the injury, the surgical intervention, and the immobilization time ranging from 6 to 9 weeks implies significant biomechanical alterations. These consequences are reflected by decreased range of motion of the ankle joint, soft tissue impairments, proprioception, and loss muscle strength, which indirectly affects functional activities such as walking, balance, jumping, and running [10-13].

A meta-analysis researching the time course of physical recovery after ankle fracture with data from 23 studies concludes that adults, on average, recovered rapidly from activity limitation in the first 3 months after fracture, improved little between 3 and 6 months, and stabilized, without reaching full recovery, at 24 months [5].

Gait analysis is of particular importance in clinical practice, whether to evaluate a rehabilitation process or after surgery [14]. As part of this analysis, spatial-temporal parameters provide us with a considerable amount of information on relevant and functional aspects. It describes quantitatively the main gait events, and therefore reflects the patient's ability to meet the general requirements of gait, i.e., single stance, double stance, and swing leg forward movement [15].

Currently, motion capture systems based on optical technology allow to quantitatively assess the functional limitations that exist in patients with musculoskeletal pathology of the lower extremity $[9,16]$. These systems where we are analyzed kinematic values and spatial-temporal parameters are useful for both diagnosis and treatment and reinforce confidence in the decision-making process of professionals [17]. On the other hand, despite being the "Gold Standard" in gait analysis, are not easily portable, require lengthy calibration and data analysis must be performed by qualified professionals. In addition, they are expensive, which may limit their accessibility for both clinical and research fields [18].

As an alternative for motion analysis, the use of low-cost inertial sensors (IMUs) has been shown to have a measurement efficiency similar to higher technologies systems [19-22]. These sensors, which integrate accelerometers, gyroscopes, and magnetometers, allow measurements to be taken outside the laboratory and generally have intuitive software that is easy for the professional to use on a daily basis [23].

The number of IMUs and their distribution in the different body segments can provide us with very complete information on the biomechanics of the human body. However, as shown by several studies in healthy subjects [24] and with lower limb pathology [25], the individual use of a single IMU placed on the lumbar spine, allows us to obtain reliable information based on trunk acceleration and angular velocity algorithms to estimate the spatial-temporal gait parameters [19, 26, 27].

Finally, a recent meta-analysis provides encouraging results in terms of the validity and reliability of IMUs for measuring step/stride length and time. However, it warns of the importance of a strict assessment protocol for symmetry and variability in gait analysis [22]. 
The aim of this study is 1) to assess in patients with ankle fracture 6 months after surgery the differences between the operated and healthy ankle in spatial-temporal gait parameters and dynamic plantar pressures 2) to study the possible relationship with clinical measurements and scales. 3) to assess the reliability of a single inertial sensor, placed on the lumbar spine, in this population.

\section{Methods}

\section{Type of study}

This cross-sectional study was carried out in the movement analysis laboratory of the University Schools of Physiotherapy and Speech Therapy Gimbernat-Cantabria attached to the University of Cantabria.

\section{Participants}

The population was composed of Twenty-two participants (ten women/twelve men) who underwent surgery after a bimalleolar ankle fracture at the Trauma Unit of the University Hospital "Marqués de Valdecilla" (UHMV) in Santander. The surgical technique used was open reduction and internal fixation (ORIF) and the time elapsed from injury to surgery was $4.8 \pm 7.6$ days. After the immobilization period (3.4 \pm 1.2 weeks), progressive and variable rehabilitation was carried out depending on the individual improvement of each case ( $13 \pm 2.4$ weeks) 5 days a week by the physiotherapy service of the UHMV. Inclusion criteria were established as 6 months after surgery and age between 18 and 55 years. Patients with previous surgery on the lower limb, bilateral ankle involvement, neurological and rheumatic pathology were excluded.

Subjects were selected through medical records registered at the UHMV and with the collaboration of the Trauma Unit. After the Informed Consent was approved in writing by the Cantabrian Research Ethics Committee (CEIC) (reference: 2017.072), they were invited to participate by telephone or email, where they were informed of the objective of the study and the procedure to be followed for its realization.

\section{Procedure}

Data collection was carried out in a single individual visit and after a brief explanation of the procedure to be followed, the Informed Consent was signed. Sociodemographic and clinical information was previously collected. The clinical data collected were firstly the American Orthopaedic Foot and Ankle Society (AOFAS) Ankle-Hindfoot score [28] and the Olerud Molander Ankle Score (OMAS) [29] questionnaires, which assess the functional status of the patients. Subsequently, physical examination of both legs was performed by anthropometric measurement, bimalleolar/calf perimeters, ankle dorsiflexion range of motion (ADF ROM), and hip abductor (ABD)/adductor (ADD) muscle strength. The protocol performed for the clinical measurements was described in detail in our previous study [13].

The gait cycle (GC) analysis was performed with the subject barefoot on a walkway $8 \mathrm{~m}$ long and $2.5 \mathrm{~m}$ wide where he had to perform 4 laps $(32 \mathrm{~m})$ at his normal walking speed. We considered normal speed to be the speed previously preferred by each subject after a brief trial at different speeds following the 
recommendations of some authors for gait analysis on level ground [30]. Two valid trials were collected for each subject, discarding in the processing the first and last step of each lap. For gait analysis, a wireless inertial sensor system BTS G-WALK (BTS Bioengineering S.p.A. Italy) weighing $37 \mathrm{~g}$ and measuring $70 \times 40 \times 18 \mathrm{~mm}$ was used, placed by means of a semi-elastic belt at the level of the fifth lumbar vertebra (L5). This inertial system is equipped with 4-Sensor Fusion technology that integrates a triaxial accelerometer (16 bits/axis, $\pm 8 \mathrm{~g}$ ), a triaxial magnetometer (13bits, $\pm 1200 \mathrm{uT}$ ), a triaxial gyroscope (16 bits/axis, $\pm 250 \%$ s), and a GPS receiver. All data were collected at a frequency of $100 \mathrm{~Hz}$ and transmitted through a Bluetooth 3.0 connection to the computer. A specific software (BTS G-Studio) allows processing the information and calculating the spatial-temporal gait parameters and the percentage of symmetry for these parameters between both limbs. The exact algorithms of the G-WALK are unknown and are part of the internal organization of the BTS company. However, some studies validate its use in different populations $[21,26,27]$.

The general spatial-temporal parameters collected were cadence (strides/min), speed $(\mathrm{m} / \mathrm{s})$ and stride length $(\mathrm{m})$. Bilateral spatial-temporal parameters (leg differences expressed as a percentage of the gait cycle) were step length (\% stride length), stance phase (\%GC), swing phase (\%GC), double support (\%GC), single support (\%GC), and propulsion $\left(\mathrm{m} / \mathrm{s}^{2}\right.$, the difference in anterior/posterior acceleration of the body barycenter between toe-off to heel strike events) [22].

Finally, complementary to the spatial-temporal gait parameters, we collected information on dynamic plantar pressure, the variables analyzed being step time ( $\mathrm{ms}$ ) and mean plantar pressure $(\mathrm{kPa})$ for each leg. The tool used was a single P-WALK pressure platform (BTS Bioengineering S.p.A. Italy) with dimensions of $640 \times 740 \times 8 \mathrm{~mm}$ (acquisition rate of $250 \mathrm{~Hz}$ ) and the software used was the same as for the inertial sensor (BTS G-Studio). By using the same software (different acquisition rate) the devices cannot be synchronized and the P-Walk data were subsequently collected to the G-Walk data. The pressure platform was placed in the middle part $(4 \mathrm{~m})$ of the walkway. In this way, for step normalization, we ensured that at least three steps were taken before stepping on the platform. Five steps were taken with each leg during a continuous gait cycle. Studies in healthy people with different pathologies showed a moderate-high reliability (ICC >0.7) in their recordings with pressure platforms [31, 32].

\section{Statistical analysis}

Firstly, participants were classified according to their operated and healthy ankle. Sociodemographic and clinical variables were described. For categorical variables, percentages with their corresponding $95 \%$ confidence intervals $(95 \% \mathrm{Cl})$ were estimated, and for continuous variables, means were estimated with their standard deviation or, if they did not follow a normal distribution, their median and range. The Shapiro-Wilk test was performed to analyze the normality of the variables.

In each of the two ankles (operated/healthy), the results of the different variables were analyzed and the difference between them was obtained using the Student's t-test for paired samples (expressed as mean difference) or its equivalent non-parametric Wilcoxon Matched-Pairs Signed Ranks Test (expressed with the typed Z-value in order to be able to make the comparison with that of a standardized normal 
distribution). Likewise, the effect size was calculated using Cohen's $d$ or Hodges' $\mathrm{g}$ whose values are quantified as follows: 0.2 small, 0.5 medium, and 0.8 large [33].

The relationship between clinical measurements and functional scales with gait parameters and dynamic plantar pressures were analyzed using Pearson's correlation coefficient $(r)$ or Spearman's rank correlation (Rho) (non-parametric). A multiple linear regression analysis ( $r 2)$, expressed together with the F-statistic value, was then performed with the variables that showed a significant correlation to determine the extent to which clinical measurements or functional scale scores could predict gait analysis results.

Intra-session test-retest reliability of spatial-temporal gait parameters measured with the $\mathrm{G}$ walk was calculated using two valid tests. For relative reliability, an intraclass correlation coefficient model $\left(\mathrm{ICC}_{2,1}\right)$ with a $95 \% \mathrm{Cl}$ was used following the recommendations described in the literature [34]. The ICC values were classified as follows: excellent ( 0.90 to 1.00$)$, high ( 0.70 to 0.89 ), moderate ( 0.50 to 0.69 ) and low $(<0.50)$ [35]. Absolute reliability was obtained with the standard error of measurement (SEM) calculated as $S E M=S D * \sqrt{ }(1-I C C)[36]$. The $S E M$ values were expressed in the same units as the mean value to facilitate extrapolation of the results to other individuals.

Finally, Bland-Altman plots with $95 \%$ limits of agreement (LoA; mean differences \pm 1.96 SD) were generated to visualize the degree of agreement between the measurements reported. Systematic error (bias) was obtained using the mean of the differences.

GPower 3.1 statistical software was used to calculate the minimum sample size necessary to detect significant results. Based on the effect size estimate $(d=0.7)$ from previous studies [37, 38], a sample size of nineteen participants was considered necessary to detect differences between the legs (power $=80 \%$; $a$ $=0.05$ ). Twenty-two patients were recruited to allow for a dropout rate of $10 \%$.

Statistical analysis of the data was performed using SPSS 20.0 software (Statistical Product and Service Solutions IBM SPSS Statistics 19.0 2010).

\section{Results}

A total of twenty-two patients participated in the present study. The mean age was $43.5 \pm 10.2$ years with ages ranging from 21 to 55 years. Table 1 describes the demographic and anthropometric characteristics and functional status of the ankle. 
Table 1

Demographic, anthropometric, and functional characteristics of subjects with ankle fractures 6 months after surgery.

\begin{tabular}{|lll|}
\hline Type $(\mathbf{n = 2 2})$ & Mean \pm SD & $\mathbf{9 5 \%} \mathbf{C l}$ \\
\hline Age $($ years $)$ & $43.5 \pm 10.2$ & $39.0 ; 48.0$ \\
\hline Height $(\mathrm{cm})$ & $169.3 \pm 9.5$ & $164.8 ; 173.7$ \\
\hline Weight $(\mathrm{kg})$ & $77.8 \pm 16.6$ & $69.9 ; 85.5$ \\
\hline Operated Limb Length $(\mathrm{cm})$ & $85.6 \pm 5.9$ & $82.9 ; 88.2$ \\
\hline Healthy Limb Length $(\mathrm{cm})$ & $85.6 \pm 5.9$ & $82.9 ; 88.2$ \\
\hline AOFAS Ankle-Hindfoot score & $74.7 \pm 12.0$ & $69.4 ; 80.0$ \\
\hline OMAS & $57.0 \pm 21.6$ & $47.4 ; 66.6$ \\
\hline $\begin{array}{l}\text { SD: standard deviation; Cl: confidence interval; AOFAS: American Orthopedic Foot and Ankle Society; } \\
\text { OMAS: Olerud Molander Ankle Score }\end{array}$ & \\
\hline
\end{tabular}

The difference between the healthy and operated ankle in the spatial-temporal parameters of gait and dynamic plantar pressure showed a significant difference in step length $(-3.8 \% ; p=.009 ; d=0.61)$, stance phase $(Z=-2.9 ; p=.004 ; g=0.76)$, swing phase $(Z=-2.9 ; p=.004 ; g=0.76)$, single support $(Z=-3.0 ; p=.002$; $\mathrm{g}=0.80)$, propulsion $(0.8 \mathrm{~m} / \mathrm{s} 2 ; \mathrm{p}=.0 ; \mathrm{d}=0.62)$, step time $(-24.8 \mathrm{~ms} ; \mathrm{p}=.003 ; \mathrm{d}=0.73)$ and mean plantar pressure $(-2.2 \mathrm{kPa} ; \mathrm{p}<.001 ; \mathrm{d}=1.02)$. We also found differences in clinical measurements except for ADD strength with an effect size between $0.15<\mathrm{d}>2.30$ (Table 2) 
Table 2

Difference between the operated and healthy ankle in clinical measurements, spatial-temporal gait parameters and dynamic plantar pressure.

\begin{tabular}{|c|c|c|c|c|c|c|}
\hline & Type $(n=22)$ & $\begin{array}{l}\text { Operated } \\
\text { Ankle Mean } \\
\pm \text { SD/ } \\
\text { Median } \\
\text { (range) }\end{array}$ & $\begin{array}{l}\text { Healthy } \\
\text { Ankle Mean } \\
\pm \text { SD/ } \\
\text { Median } \\
\text { (range) }\end{array}$ & $\begin{array}{l}\text { Differences } \\
\text { between } \\
\text { ankles Mean } \\
(95 \% \mathrm{Cl}) / \mathrm{Z}^{1}\end{array}$ & $\begin{array}{l}\text { Cohen's } \\
d / \\
\text { Hedges' } \\
g\end{array}$ & $\begin{array}{l}P \\
\text { value* }\end{array}$ \\
\hline \multirow[t]{5}{*}{$\begin{array}{l}\text { Clinical } \\
\text { measurements }\end{array}$} & $\begin{array}{l}\text { Calf } \\
\text { perimeter } \\
(\mathrm{cm})^{1}\end{array}$ & $34.1(15.0)$ & 35.4 (17.0) & -2.8 & 0.76 & $.005^{\star}$ \\
\hline & $\begin{array}{l}\text { Bimalleolar } \\
\text { perimeter } \\
\text { (cm) }\end{array}$ & $25.1 \pm 2.1$ & $24.1 \pm 2.1$ & $1.0(0.8 ; 1.2)$ & 2.30 & $.001 *$ \\
\hline & $\begin{array}{l}\text { ADF ROM } \\
\text { (degrees) }\end{array}$ & $22.8 \pm 7.7$ & $35.4 \pm 5.3$ & $\begin{array}{l}-12.7(-15.1 ; \\
-10.3)\end{array}$ & 2.23 & $<.001 *$ \\
\hline & $\begin{array}{l}\text { Strength } \\
\text { ABD (\%) }\end{array}$ & $25.5 \pm 7.2$ & $29.3 \pm 8.6$ & $-3.8(-6.4 ;-1.2)$ & 0.62 & $.006^{\star}$ \\
\hline & $\begin{array}{l}\text { Strength } \\
\text { ADD (\%) }\end{array}$ & $26.3 \pm 9.1$ & $25.8 \pm 8.6$ & $0.6(-1.1 ;-2.2)$ & 0.15 & .491 \\
\hline \multirow{9}{*}{$\begin{array}{l}\text { Spatial- } \\
\text { temporal } \\
\text { parameters }\end{array}$} & $\begin{array}{l}\text { Cadence } \\
\text { (step/min) }\end{array}$ & $99.9 \pm 9.8$ & & & & \\
\hline & $\begin{array}{l}\text { Speed } \\
\text { normalized } \\
(\mathrm{m} / \mathrm{s})\end{array}$ & $0.96 \pm 0.1$ & & & & \\
\hline & $\begin{array}{l}\text { Stride } \\
\text { length (m) }\end{array}$ & $1.15 \pm 0.1$ & & & & \\
\hline & $\begin{array}{l}\text { Step length } \\
\% \text { SL }\end{array}$ & $48.1 \pm 3.1$ & $51.9 \pm 3.1$ & $-3.8(-6.7 ;-1.1)$ & 0.61 & $.009 *$ \\
\hline & $\begin{array}{l}\text { Stance \% } \\
\mathrm{GC}^{1}\end{array}$ & $63.4(20.3)$ & $67.4(17.9)$ & -2.9 & 0.76 & $.004^{\star}$ \\
\hline & $\begin{array}{l}\text { Swing \% } \\
\mathrm{GC}^{1}\end{array}$ & $36.6(20.3)$ & $32.6(17.9)$ & -2.9 & 0.76 & $.004^{\star}$ \\
\hline & $\begin{array}{l}\text { Double } \\
\text { support \% } \\
\text { GC }\end{array}$ & $15.0 \pm 4.3$ & $16 \pm 2.1$ & $-1.0(-2.8 ;-0.8)$ & 0.25 & .267 \\
\hline & $\begin{array}{l}\text { Single } \\
\text { support \% } \\
\mathrm{GC}^{1}\end{array}$ & $32.6(17.6)$ & $36.7(20.6)$ & -3.0 & 0.80 & $.002^{\star}$ \\
\hline & $\begin{array}{l}\text { Propulsion } \\
\left(\mathrm{m} / \mathrm{s}^{2}\right)\end{array}$ & $6.0 \pm 1.4$ & $5.2 \pm 1.8$ & $0.8(0.2 ; 1.2)$ & 0.62 & $.010 *$ \\
\hline
\end{tabular}




\begin{tabular}{|c|c|c|c|c|c|c|}
\hline & Type $(n=22)$ & $\begin{array}{l}\text { Operated } \\
\text { Ankle Mean } \\
\pm \text { SD/ } \\
\text { Median } \\
\text { (range) }\end{array}$ & $\begin{array}{l}\text { Healthy } \\
\text { Ankle Mean } \\
\pm \text { SD/ } \\
\text { Median } \\
\text { (range) }\end{array}$ & $\begin{array}{l}\text { Differences } \\
\text { between } \\
\text { ankles Mean } \\
(95 \% \mathrm{Cl}) / \mathrm{Z}^{1}\end{array}$ & $\begin{array}{l}\text { Cohen's } \\
\text { d/ } \\
\text { Hedges' }\end{array}$ & $\begin{array}{l}P \\
\text { value* }\end{array}$ \\
\hline \multirow{2}{*}{$\begin{array}{l}\text { Dynamic } \\
\text { plantar } \\
\text { pressure }\end{array}$} & $\begin{array}{l}\text { Step time } \\
\text { (ms) }\end{array}$ & $822.0 \pm 136$ & $\begin{array}{l}846.8 \pm \\
127.4\end{array}$ & $\begin{array}{l}-24.8(-39.9 \\
-9.6)\end{array}$ & 0.73 & $.003^{*}$ \\
\hline & $\begin{array}{l}\text { Average } \\
\text { plantar } \\
\text { pressure } \\
(\mathrm{kPa})\end{array}$ & $52.8 \pm 4.0$ & $55.0 \pm 3.8$ & $-2.2(-3.2 ;-1.2)$ & 1.02 & $<.001 *$ \\
\hline \multicolumn{7}{|c|}{$\begin{array}{l}\text { SD: Standard deviation; Cl: Confidence interval; ADF ROM: Ankle Dorsiflexion Range of Movement; } \\
\text { ABD: Hip abductor muscle; ADD: Hip adductor muscle; ROM: Range of movement; GC: Gait cycle; SL: } \\
\text { Stride length; Cohen's } d \text { : Size effect; Hedges' g. Size effect (non-parametric); }{ }^{1} \text { Wilcoxon Matched- } \\
\text { Pairs Signed Ranks Test (non-parametric; expressed with the typed Z-value); * Significance level P < } \\
.05\end{array}$} \\
\hline
\end{tabular}


TABLE 3

Correlation between clinical measurements and functional scales with the spatial-temporal gait parameters and dynamic plantar pressure in operated ankle.

\begin{tabular}{|c|c|c|c|c|c|c|}
\hline \multirow[b]{2}{*}{$\begin{array}{l}\text { Spatial-temporal gait } \\
\text { parameters }\end{array}$} & \multicolumn{6}{|c|}{ Clinical measurements and functional scales } \\
\hline & $\begin{array}{l}\text { ADF } \\
\text { ROM }\end{array}$ & $\begin{array}{l}\text { Strength } \\
\text { ABD }\end{array}$ & $\begin{array}{l}\text { Bimalleolar } \\
\text { perimeter }\end{array}$ & $\begin{array}{l}\text { Calf } \\
\text { perimeter }\end{array}$ & AOFAS & OMAS \\
\hline Cadence (step/min $)^{1}$ & $.543^{\star \star *}$ & .401 & .227 & .109 & $.540 \star \star$ & .415 \\
\hline $\begin{array}{l}\text { Speed normalized } \\
(\mathrm{m} / \mathrm{s})^{1}\end{array}$ & $.483^{\star}$ & $.499 *$ & .049 & -.057 & $.428^{\star}$ & .247 \\
\hline Stride length $(m)^{1}$ & .165 & .276 & .281 & -.049 & .247 & .083 \\
\hline Step length \% SL ${ }^{1}$ & -.001 & .231 & .804 & -.076 & .163 & .205 \\
\hline Stance $\% \mathrm{GC}^{2}$ & -.054 & -.178 & -.112 & .144 & .085 & .136 \\
\hline Swing $\% \mathrm{GC}^{2}$ & .115 & .178 & .112 & -.144 & -.085 & -.136 \\
\hline Double support \% GC ${ }^{1}$ & -.224 & -.303 & -.060 & .204 & -0.69 & .036 \\
\hline Single support $\% \mathrm{GC}^{2}$ & .327 & $.529 *$ & .058 & -.091 & .402 & .284 \\
\hline Propulsion $\left(\mathrm{m} / \mathrm{s}^{2}\right)^{1}$ & $.523 *$ & -.052 & .122 & $.447 \star$ & .407 & .261 \\
\hline Step time $(\mathrm{ms})^{1}$ & $-.543^{\star \star}$ & -.416 & -.315 & .025 & $-.454^{\star}$ & -.403 \\
\hline $\begin{array}{l}\text { Average plantar } \\
\text { pressure }(\mathrm{kPa})^{1}\end{array}$ & -.307 & $-.481^{*}$ & .154 & .351 & -.233 & -.274 \\
\hline
\end{tabular}




\section{TABLE 4}

Intra-session test-retest reliability spatial-temporal gait parameters with the inertial sensor $\mathbf{G}$ walk. Limits of Agreement (Bland-Altman analysis) and mean of the differences (bias) between two trials.

\begin{tabular}{|c|c|c|c|c|c|}
\hline & $\begin{array}{l}\text { Spaciotemporal gait } \\
\text { parameters }\end{array}$ & ICC $(95 \% \mathrm{Cl})$ & SEM $(95 \%$ IC $)$ & $\begin{array}{l}\text { LoA } \\
\text { (lower; } \\
\text { upper) }\end{array}$ & Bias \\
\hline & Cadence (step/min) & $\begin{array}{l}0.95(0.89 \\
0.97)\end{array}$ & $\begin{array}{l}2.21(0.79 \\
-3.64)\end{array}$ & $-3.91 ; 2.12$ & -0.89 \\
\hline & Speed (m/s) & $\begin{array}{l}0.97(0.93 ; \\
0.98)\end{array}$ & $\begin{array}{l}0.02(0.01 ; \\
0.05)\end{array}$ & $-0.06 ; 0.04$ & -0.01 \\
\hline & Stride length $(m)$ & $\begin{array}{l}0.98(0.97 ; \\
0.99)\end{array}$ & $\begin{array}{l}0.02(0.01 ; \\
0.03)\end{array}$ & $-0.07 ; 0.06$ & 0.01 \\
\hline \multirow[t]{6}{*}{$\begin{array}{l}\text { Operated } \\
\text { Ankle }\end{array}$} & Step length \% SL & $\begin{array}{l}0.90(0.82 \\
0.94)\end{array}$ & $\begin{array}{l}1.01(0.55 \\
1.46)\end{array}$ & $-2.17 ; 1.92$ & -0.12 \\
\hline & Stance phase \% GC & $\begin{array}{l}0.91(0.84 ; \\
0.94)\end{array}$ & $\begin{array}{l}1.43(0.75 \\
2.12)\end{array}$ & $-5.02 ; 4.51$ & -0.26 \\
\hline & Swing phase $\%$ GC & $\begin{array}{l}0.86(0.75 \\
0.91)\end{array}$ & $\begin{array}{l}1.79(1.10 \\
2.47)\end{array}$ & $-4.51 ; 5.02$ & 0.26 \\
\hline & Double support \% GC & $\begin{array}{l}0.85(0.74 ; \\
0.91)\end{array}$ & $\begin{array}{l}1.68(1.06 ; \\
2.31)\end{array}$ & $-6.80 ; 7.74$ & 0.47 \\
\hline & Single support \% GC & $\begin{array}{l}0.84(0.74 \\
0.91)\end{array}$ & $\begin{array}{l}1.82(1.17 \\
2.48)\end{array}$ & $-9.21 ; 5.63$ & -1.79 \\
\hline & Propulsion $\left(\mathrm{m} / \mathrm{s}^{2}\right)$ & $\begin{array}{l}0.90(0.83 \\
0.94)\end{array}$ & $\begin{array}{l}0.45(0.24 ; \\
0.65)\end{array}$ & $-1.79 ; 1.89$ & 0.05 \\
\hline \multirow[t]{6}{*}{$\begin{array}{l}\text { Healthy } \\
\text { Ankle }\end{array}$} & Step length \% GC & $\begin{array}{l}0.90(0.84 ; \\
0.95)\end{array}$ & $\begin{array}{l}1.01(0.55 ; \\
1.46)\end{array}$ & $-1.92 ; 2.17$ & 0.12 \\
\hline & Stance phase \% GC & $\begin{array}{l}0.94(0.89 ; \\
0.96)\end{array}$ & $\begin{array}{l}1.12(0.46 ; \\
1.77)\end{array}$ & $-2.96 ; 4.50$ & 0.77 \\
\hline & Swing phase $\%$ GC & $\begin{array}{l}0.92(0.86 \\
0.95)\end{array}$ & $\begin{array}{l}1.29(0.63 \\
1.95)\end{array}$ & $-4.50 ; 2.96$ & -0.77 \\
\hline & Double support \% GC & $\begin{array}{l}0.84(0.73 ; \\
0.90)\end{array}$ & $\begin{array}{l}1.06(0.68 ; \\
1.44)\end{array}$ & $-5.57 ; 7.83$ & 1.13 \\
\hline & Single support \% GC & $\begin{array}{l}0.84(0.73 ; \\
0.91)\end{array}$ & $\begin{array}{l}1.90(1.22 ; \\
2.58)\end{array}$ & $-7.59 ; 8.17$ & 0.29 \\
\hline & Propulsion $\left(\mathrm{m} / \mathrm{s}^{2}\right)$ & $\begin{array}{l}0.95(0.92 ; \\
0.97)\end{array}$ & $\begin{array}{l}0.41(0.15 \\
0.68)\end{array}$ & $-1.89 ; 1.20$ & -0.34 \\
\hline
\end{tabular}

Cl. confidence interval; ICC. intraclass correlation coefficient; SEM. standard error of the measurement; LoA: limits of agreement; Bias: mean of the differences 
Correlation analysis between clinical measurements and dynamic plantar pressure with spatial-temporal gait parameters in the operated ankle showed statistically significant results $\left({ }^{*} p<.050 ;{ }^{*} p<<.010\right)$ and a moderate to large effect size (Table 3). Multiple linear regression analysis showed that both ADF ROM and $A B D$ strength scores can explain the variability of gait analysis results between 24 and $48 \%$. Specifically, cadence increased with increasing ADF ROM $r=0.543 * \star(F(1,21)=8.4, r 2=0.30, p=.009)$; speed increased with increasing ADF ROM $r=0.483^{*}$ and increasing ABD strength $r=0.499 *(F(1,21)=6.6$, $r 2=0.25, p=.018)$; single support increased with greater $A B D$ strength $R h o=0.529 *(F(1,21)=6.3, r 2=$ $0.24, p=.020)$, propulsion was greater the higher the ADF ROM $r=0.523^{*}$ and calf perimeter $r=0.447^{*}(F$ $(1,21)=10, r 2=0.46, p=.001)$. Correlation was negative between step time and ADF ROM $r=-0.543^{\star \star}(F$ $(1,21)=8.4, r 2=0.30, p=.009)$ as well as between mean plantar pressure and ABD strength $r=-0.481 *(F$ $(1,21)=6.2, r 2=0.23, p=.023)$. Finally, with respect to the AOFAS scores, the correlation was positive between cadence $\left(r=0.540^{\star *}\right)$ and speed $\left(0.428^{*}\right)$, and negative with step time $\left(-0.454^{\star}\right)$. Multiple linear regression analysis showed that only cadence could explain the variability of the AOFAS score by $29 \%$ (F $(1,21)=8.2, r 2=0.29, p=.009)$.

The intra-session test-retest reliability analysis, including $\mathrm{ICC}_{2,1}$ and SEM values, are shown in Table 4. Excellent relative reliability scores $(0.95 \leq \mathrm{ICC} \leq 0.98)$ were found for the general parameters of gait analysis, as well as low values of SEM. For the bilateral parameters, a good to excellent ICC score was found with values between 0.84 and 0.95 . The worst SEM values were for the single support of the operated and healthy ankle with values of $1.82 \% \mathrm{GC}$ and $1.90 \% \mathrm{GC}$ respectively.

Figure 1 show the Bland-Altman plots comparing the results of the spatial-temporal gait parameters. The horizontal line represents the mean of the differences, while the dotted lines represent the confidence interval. The Bland-Altman plot analysis showed an excellent degree of agreement between measurements for speed (bias $=-0.01$; LoA $=-0.06 ; 0.04$ ) and stride length (bias $=0.01 ; \operatorname{LoA}=-0.07 ; 0.06$ ). Single support (bias $=-1.79$; LoA $=-9.21 ; 6.63$ ) in the operated ankle and double support (bias = 1.13 ; LoA= $-5.57 ; 7.83)$ in the healthy ankle showed the lowest degrees of agreement. The mean error and limits of agreement for the remaining variables are reported in Table 4.

\section{Discussion}

Our study aimed to identify the functional consequences in subjects with bimalleolar ankle fractures 6 months after surgery. To quantify these limitations, we performed an analysis of spatial-temporal gait parameters and plantar pressures, as well as measuring clinical parameters and functional scales. We evaluated the degree of correlation between these variables and their clinical implication. Finally, we analyzed the reliability and reproducibility of a single inertial sensor, placed on the lumbar spine, in our study population.

In terms of the results obtained in spatial-temporal gait parameters, we found differences between the operated and healthy ankle in all parameters except double support. Our findings are consistent with a study performed in thirty subjects with ankle fractures, where they found these differences between the 
operated and healthy ankle after a 12-week period of specific rehabilitation in step time, step length, swing phase, stance phase, and single support [39]. Segal et al [7] in their study of forty-one subjects with ankle fracture, found in the bimalleolar fracture group $(n=15)$ differences between the two ankles in step length $(-29.2 \% \mathrm{SL})$ and single support $(-15.9 \% \mathrm{GC})$. In our patients, we also found this asymmetry in stride length (-3.8\% SL) and individual support (-4.1\% GC), although the difference was not as large. In addition, the speed was only $0.48 \mathrm{~m} / \mathrm{s}$, very different from that found in our study $(0.96 \mathrm{~m} / \mathrm{s})$. The differences in the results could be justified by the period of the measurements, as the study was performed from the 12th week after surgery, just when the weight-bearing on the operated ankle was allowed.

Specific rehabilitation after a prolonged period of immobilization is important, as not only muscle impairment [40], but functional capacity as measured by gait analysis or stair climbing may also be impaired $[7,10,39]$. Some authors even find that one year after surgery, the spatial-temporal gait parameters are still not normalized $[8,9,16]$. Van Hoeve et al [16], in their study conducted on thirty-three subjects 18 months after surgery, in addition to gait alterations, found a negative correlation between speed and ankle flexion/extension ROM. This is consistent with our study where in addition to ADF ROM we found this correlation of gait speed with $A B D$ strength. Furthermore, we observed that ADF ROM DF and $A B D$ strength had a moderate association with cadence, speed, and stride length and predicted almost $30 \%$ of the variability of their values.

ADF ROM is one of the most studied variables after ankle injury [41]. In our research, the measurement was performed before the gait analysis to correlate it with the different parameters. Nevertheless, this ankle motion quantification system had excellent intra- and inter-test reliability [42]. Studies using 3D kinematic analysis systems show the restrictions of the ADF ROM during the different phases of gait [9, 16]. Using a methodology similar to ours, Nilsson et al [43], in a sample of one hundred and ten patients who underwent surgery after an ankle fracture, obtained the same results in the measurement of the ADF ROM at 6 months. Despite rehabilitation efforts to improve ankle motion, short- and medium-term studies conclude that the gain is only between 6 and 12\% [41]. This low gain in ROM is a major barrier to acquiring pre-injury status.

The study of dynamic plantar pressure provides very precise information on the type of tread during gait. In our investigation, in addition to significant differences between both ankles in mean plantar pressure and step time, we found a negative correlation with ADF ROM $(r=-0.543 ; p<.010)$ and ABD strength $(r=$ $-0.481 ; p<.050)$ respectively. A study performed on forty patients who underwent surgery after ankle fracture where they evaluate the asymmetry of plantar pressures during gait, concludes that, in addition to this asymmetric pattern between both limbs, they tended to exert greater pressure on the lateral forefoot [38]. Although in our study we did not analyze plantar pressures by zones and only obtained the global value of the gait, other authors reported similar results to those found above [44-46]. These results could be interpreted as a protective factor when performing the step with the operated ankle. Also, the increased ankle stiffness in dorsiflexion causes excessive heel elevation in the final stance phase of the gait cycle. 
The muscle atrophy that can be evidenced in the calf diameter could influence the gait pattern of patients with ankle fracture. In our study, only 24 weeks after surgery and after a period of immobilization and rehabilitation without load, the muscle of the operated ankle has a smaller diameter $(-1.3 \mathrm{~cm} ; \mathrm{p}=.002)$ compared to the healthy ankle. In addition, we found a positive and significant correlation between calf diameter and propulsion ( $r=.447)$ and in conjunction with ADF ROM $(r=.523)$ could predict $46 \%$ of the propulsion outcome. Human studies quantifying the effect of disuse on muscle morphology show that in only 8 weeks of immobilization the cross-sectional area measured with MRI shows decreases of $19 \%$ and $24 \%$ in the anterior and posterior calf muscle compartments [40].

A meta-analysis which studied the long-term prognosis in the improvement of function in patients operated after an ankle fracture, tells us that the improvement in the first 6 months is rapid but incomplete, where only $78 \%$ of the function is recovered [5]. In our study, we even found worse results on the OMAS subjective functional scale $(57.0 \pm 21.6)$, which was used in most of the studies included in the meta-analysis. In addition, we found that higher cadence and speed and shorter step time correlated with better AOFAS scores. However, this correlation was not found with the OMAS scale. This is in line with the findings of other studies in which this correlation also did not exist $[39]$ or was weak $[8,9]$.

Among the purposes of this study was to assess the reliability of a single inertial sensor, placed on the lumbar spine, in this population.

In our results we found high to excellent intra-session test-retest reliability, with ICC scores between 0.84 and 0.98; the worst ICC values were obtained for the variables single support and double support. Speed, stride length and propulsion obtained the lowest SEM scores. The degree of agreement between the two trials was excellent for the same parameters, with bias values close to 0 and a small range in LoA. Our results are consistent with those found by De Ridder et al [26] in a group of thirty healthy subjects where they obtained high to excellent reliability values $(0.85<\mid C C>0.99)$ in the spatial-temporal gait parameters after five valid trials. Bravi et al [25] in their study on twenty subjects with lower limb pathology found moderate to excellent intra-session test-retest reliability $(0.59<\mid \mathrm{CC}>0.95)$ with the lowest values corresponding to the phases of the gait cycle. In our study, we did not find such low ICC values in the gait phases. However, we found higher SEM in the swing phase, single support, and double support of the operated ankle, although they only represent less than $5 \%$ of the SEM. In reference to the limits of agreement and the estimated bias, the single support of the operated ankle obtained the worst precision (bias $=-1.79$; LoA $=-9.21 ; 5.63$ ), probably justified by the presence of three outliers.

Although in our study we did not use a Gold Standard system for the concurrent validity of the sensor, there are already studies in the literature in healthy and pathological populations that demonstrate its advantages and limitations compared to other measuring devices such as GAITRite ${ }^{\circledR}[21,26,27]$, optoelectronic motion capture system (mocap) [25] and other IMUs [47, 48]. A recent systematic review and meta-analysis support the above results by highlighting good to excellent validity and reliability for time/step length and stride measures, however, spatiotemporal asymmetry measures present inconsistent validity and reliability results that could be biased by the quality of the studies and the 
difference in protocols used for gait analysis [22]. Among the explanations suggested as possible causes for the lack of reliability in some parameters are the algorithms used for event detection (heel contact and toe-off) $[21,26]$ or the limited space used by the reference systems which makes data acquisition difficult [25].

Our study has some limitations. Firstly, the characteristics of a cross-sectional study. However, we believe the need for this type of study, carried out 6 months after surgery, is necessary because of the importance of an objective identification of functional problems that can guide a more specific rehabilitation. Secondly, we have a relatively small sample size and no control group. Finally, we would have liked a more sophisticated gait analysis system, such as an instrumented walkway with pressure platforms or a 3D camera system. These systems would allow us to contrast the information provided by the sensor and thus provide more detailed reliability of the records. Likewise, we have previously commented on the agreement between the G-WALK and other measurement systems (concurrent validity) provided by different authors in different study populations [21, 25-27].

Future research should continue along the lines of what has been done so far, refining assessment protocols and study design as technology advances.

\section{Conclusion}

Patients with bimalleolar ankle fractures present remarkable functional alterations 6 months after surgery. Analysis of spatial-temporal gait parameters and plantar pressures show an evident asymmetry between both limbs; likewise, they correlate moderately with clinical measures such as ADF ROM and ABD strength, as well as with the AOFAS scale. The $G$ Walk inertial sensor can be considered an easy-touse and reliable gait analysis system for the healthcare professional. Although the data are not comparable in terms of accuracy with other more sophisticated systems, its use could be justified in the clinical setting, but caution should be exercised in the interpretation of the results regarding the identification of gait phases.

\section{Abbreviations}

AOFAS: American Orthopaedic Foot and Ankle Society

OMAS: Olerud Molander Ankle Score

ORIF: Open reduction and internal fixation

IMU: Inertial Measurement Unit

HUMV: University Hospital "Marques de Valdecilla"

GC: Gait cycle 
SL: Stride length

ADF ROM: ankle dorsiflexion range of motion

ABD: Abductor

ADD: Adductor

ICC: Intraclass correlation coefficient

SEM: Standard error of measurement

LoA: limits of agreement

\section{Declarations}

\section{Ethical approval and consent to participate}

We obtained signed informed consent from all participants, and the study design was approved by the Cantabrian Research Ethics Committee (CEIC) (reference: 2017.072).

\section{Consent for publication}

All individuals included in the study gave signed consent for publication. All authors consented to publication.

\section{Availability of data and materials}

All data can be made available from the corresponding author upon reasonable request.

\section{Conflicting Interests}

The authors declare that they have no conflicts of interest.

\section{Funding}

This research received no specific grant from any funding agency in the public, commercial, or not-forprofit sectors.

\section{Authors' contributions}


MFG conceived the idea for the study, analyzed and interpreted data, statistics, carried out measurements, and was the major contributor in writing the manuscript. DSG conceived the idea for the study, carried out measurements, and contributed to writing the manuscript. All authors contributed to the proof-reading of the manuscript and have approved the final article.

\section{Acknowledgements}

The authors would like to acknowledge the excellent cooperation of the patients in this project. We thank Vanesa Pérez and David Ventura for their continuous support during this project.

\section{References}

1. Shibuya N, Davis ML, Jupiter DC. Epidemiology of Foot and Ankle Fractures in the United States: An Analysis of the National Trauma Data Bank (2007 to 2011), J Foot Ankle Surg 53 (2014) 606-8. https://doi.org/10.1053/j.jfas.2014.03.011.

2. Elsoe R, Ostgaard SE, Larsen P. Population-based epidemiology of 9767 ankle fractures, Foot Ankle Surg. Off J Eur Soc Foot Ankle Surg. 2018;24:34-9. https://doi.org/10.1016/j.fas.2016.11.002.

3. Juto H, Nilsson H, Morberg P. Epidemiology of Adult Ankle Fractures: 1756 cases identified in Norrbotten County during 2009-2013 and classified according to AO/OTA, BMC Musculoskelet. Disord. 2018;19:441. https://doi.org/10.1186/s12891-018-2326-x.

4. Stufkens SAS, van den Bekerom MPJ, Kerkhoffs GMMJ, Hintermann B, van Dijk CN. Long-term outcome after 1822 operatively treated ankle fractures: A systematic review of the literature. Injury. 2011;42:119-27. https://doi.org/10.1016/j.injury.2010.04.006.

5. Beckenkamp PR, Lin C-WC, Chagpar S, Herbert RD, van der Ploeg HP, Moseley AM. Prognosis of physical function following ankle fracture: a systematic review with meta-analysis. J Orthop Sports Phys Ther. 2014;44:841-51. https://doi.org/10.2519/jospt.2014.5199. B2.

6. Elbaz A, Mor A, Segal G, Bar D, Monda MK, Kish B, Nyska M, Palmanovich E. Lower Extremity Kinematic Profile of Gait of Patients After Ankle Fracture: A Case-Control Study, J. Foot Ankle Surg. Off Publ Am Coll Foot Ankle Surg. 2016;55:918-21. https://doi.org/10.1053/j.jfas.2016.04.004.

7. Segal G, Elbaz A, Parsi A, Heller Z, Palmanovich E, Nyska M, Feldbrin Z, Kish B. Clinical outcomes following ankle fracture: a cross-sectional observational study. J Foot Ankle Res. 2014;7:50. https://doi.org/10.1186/s13047-014-0050-9.

8. Losch A, Meybohm P, Schmalz T, Fuchs M, Vamvukakis F, Dresing K, Blumentritt S, Stürmer KM. [Functional results of dynamic gait analysis after 1 year of hobby-athletes with a surgically treated ankle fracture], Sportverletz. Sportschaden Organ Ges. Orthopadisch-Traumatol Sportmed. 2002;16:101-7. https://doi.org/10.1055/s-2002-34750.

9. Wang R, Thur CK, Gutierrez-Farewik EM, Wretenberg P, Broström E. One year follow-up after operative ankle fractures: a prospective gait analysis study with a multi-segment foot model. Gait Posture. 2010;31:234-40. https://doi.org/10.1016/j.gaitpost.2009.10.012. 
10. Shaffer MA, Okereke E, Esterhai JL, Elliott MA, Walter GA, Yim SH, Vandenborne K. Effects of Immobilization on Plantar-Flexion Torque, Fatigue Resistance, and Functional Ability Following an Ankle Fracture. Phys Ther. 2000;80:769-80. https://doi.org/10.1093/ptj/80.8.769.

11. Hong CC, Roy SP, Nashi N, Tan KJ. Functional outcome and limitation of sporting activities after bimalleolar and trimalleolar ankle fractures. Foot Ankle Int. 2013;34:805-10. https://doi.org/10.1177/1071100712472490.

12. Dudek K, Drużbicki M, Przysada G, Śpiewak D. Assessment of standing balance in patients after ankle fractures. Acta Bioeng Biomech Wroc Univ Technol. 2014;16:59-65.

13. Salas-Gómez D, Fernández-Gorgojo M, Sanchez-Juan P, Bercero EL, Isabel Perez- Núñez M, Barbado D. Quantifying balance deficit in people with ankle fracture six months after surgical intervention through the Y-Balance test, Gait Posture. (2020) S0966636220306287. https://doi.org/10.1016/j.gaitpost.2020.10.037.

14. Rosenbaum D, Macri F, Lupselo FS, Preis OC. Gait and function as tools for the assessment of fracture repair - the role of movement analysis for the assessment of fracture healing, Injury 45 Suppl 2 (2014) 39-43. https://doi.org/10.1016/j.injury.2014.04.007.

15. Baker R. Gait analysis methods in rehabilitation. J NeuroEngineering Rehabil. 2006;3:4. https://doi.org/10.1186/1743-0003-3-4.

16. van Hoeve S, Houben M, Verbruggen JPAM, Willems P, Meijer K, Poeze M. Gait analysis related to functional outcome in patients operated for ankle fractures. J Orthop Res Off Publ Orthop Res Soc. 2019;37:1658-66. https://doi.org/10.1002/jor.24071.

17. Wren TAL, Tucker CA, Rethlefsen SA, Gorton GE, Õunpuu S. Clinical efficacy of instrumented gait analysis: Systematic review 2020 update, Gait Posture. 80 (2020) 274-279. https://doi.org/10.1016/j.gaitpost.2020.05.031.

18. Simon SR. Quantification of human motion: gait analysis-benefits and limitations to its application to clinical problems. J Biomech. 2004;37:1869-80. https://doi.org/10.1016/j.jbiomech.2004.02.047.

19. Bugané F, Benedetti MG, Casadio G, Attala S, Biagi F, Manca M, Leardini A. Estimation of spatialtemporal gait parameters in level walking based on a single accelerometer: Validation on normal subjects by standard gait analysis. Comput Methods Programs Biomed. 2012;108:129-37. https://doi.org/10.1016/j.cmpb.2012.02.003.

20. Al-Amri M, Nicholas K, Button K, Sparkes V, Sheeran L, Davies JL. Inertial Measurement Units for Clinical Movement Analysis: Reliability and Concurrent Validity, Sensors 18 (2018). https://doi.org/10.3390/s18030719.

21. Vítečková S, Horáková H, Poláková K, Krupička R, Rưžička E, Brožová H. Agreement between the GAITRite ${ }^{\circledR}$ System and the Wearable Sensor BTS G-Walk® for measurement of gait parameters in healthy adults and Parkinson's disease patients. PeerJ. 2020;8:e8835. https://doi.org/10.7717/peerj.8835.

22. Kobsar D, Charlton JM, Tse CTF, Esculier J-F, Graffos A, Krowchuk NM, Thatcher D, Hunt MA. Validity and reliability of wearable inertial sensors in healthy adult walking: a systematic review and meta- 
analysis. J NeuroEngineering Rehabil. 2020;17:62. https://doi.org/10.1186/s12984-020-00685-3.

23. Caldas R, Mundt M, Potthast W, Buarque de Lima Neto F, Markert B. A systematic review of gait analysis methods based on inertial sensors and adaptive algorithms. Gait Posture. 2017;57:204-10. https://doi.org/10.1016/j.gaitpost.2017.06.019.

24. Zijlstra W, Hof AL. Assessment of spatio-temporal gait parameters from trunk accelerations during human walking. Gait Posture. 2003;18:1-10. https://doi.org/10.1016/s0966-6362(02)00190-x.

25. Bravi M, Gallotta E, Morrone M, Maselli M, Santacaterina F, Toglia R, Foti C, Sterzi S, Bressi F, Miccinilli S. Concurrent validity and inter trial reliability of a single inertial measurement unit for spatial-temporal gait parameter analysis in patients with recent total hip or total knee arthroplasty. Gait Posture. 2020;76:175-81. https://doi.org/10.1016/j.gaitpost.2019.12.014.

26. De Ridder R, Lebleu J, Willems T, De Blaiser C, Detrembleur C, Roosen P. Concurrent Validity of a Commercial Wireless Trunk Triaxial Accelerometer System for Gait Analysis, J Sport Rehabil 28 (2019). https://doi.org/10.1123/jsr.2018-0295.

27. Park G, Woo Y. Comparison between a center of mass and a foot pressure sensor system for measuring gait parameters in healthy adults. J Phys Ther Sci. 2015;27:3199-202. https://doi.org/10.1589/jpts.27.3199.

28. The American Orthopedic Foot and Ankle Score (AOFAS), Code Technol. We Collect Orthop. Patient Outcomes. (2017). https://www.codetechnology.com/american-orthopedic-foot-ankle-score-aofas/ (accessed February 23, 2017).

29. Olerud $\mathrm{C}$, Molander $\mathrm{H}$. A scoring scale for symptom evaluation after ankle fracture. Arch Orthop Trauma Surg Arch Orthopadische Unf-Chir. 1984;103:190-4. https://doi.org/10.1007/BF00435553.

30. Yang S, Li Q, Inertial Sensor-Based Methods in Walking Speed Estimation: A Systematic Review, Sensors. 12 (2012) 6102-6116. https://doi.org/10.3390/s120506102.

31. Izquierdo-Renau M, Pérez-Soriano P, Ribas-García V, Queralt A. Intra and intersession repeatability and reliability of the S-Plate® pressure platform. Gait Posture. 2017;52:224-6. https://doi.org/10.1016/j.gaitpost.2016.12.001.

32. Ramachandra P, Maiya AG, Kumar P. Test-Retest Reliability of the Win-Track Platform in Analyzing the Gait Parameters and Plantar Pressures During Barefoot Walking in Healthy Adults. Foot Ankle Spec. 2012;5:306-12. https://doi.org/10.1177/1938640012457680.

33. Cohen J. Statistical power analysis for the behavioral sciences. 2nd ed. Hillsdale: L. Erlbaum Associates; 1988.

34. Weir JP. Quantifying test-retest reliability using the intraclass correlation coefficient and the SEM. J Strength Cond Res. 2005;19:231-40. https://doi.org/10.1519/15184.1.

35. Kottner J, Audige L, Brorson S, Donner A, Gajewski BJ, Hróbjartsson A, Roberts C, Shoukri M, Streiner DL. Guidelines for Reporting Reliability and Agreement Studies (GRRAS) were proposed. Int J Nurs Stud. 2011;48:661-71. https://doi.org/10.1016/j.jjnurstu.2011.01.016.

36. de Vet HCW, Terwee CB, Knol DL, Bouter LM. When to use agreement versus reliability measures. J Clin Epidemiol. 2006;59:1033-9. https://doi.org/10.1016/j.jclinepi.2005.10.015. 
37. Albin SR, Koppenhaver SL, Marcus R, Dibble L, Cornwall M, Fritz JM. Short-term Effects of Manual Therapy in Patients After Surgical Fixation of Ankle and/or Hindfoot Fracture: A Randomized Clinical Trial. J Orthop Sports Phys Ther. 2019;49:310-9. https://doi.org/10.2519/jospt.2019.8864.

38. Becker HP, Rosenbaum D, Kriese T, Gerngross H, Claes L. Gait asymmetry following successful surgical treatment of ankle fractures in young adults, Clin. Orthop. (1995) 262-269.

39. Suciu O, Onofrei RR, Totorean AD, Suciu SC, Amaricai EC. Gait analysis and functional outcomes after twelve-week rehabilitation in patients with surgically treated ankle fractures. Gait Posture. 2016;49:184-9. https://doi.org/10.1016/j.gaitpost.2016.07.006.

40. Stevens JE, Walter GA, Okereke E, Scarborough MT, Esterhai JL, George SZ, Kelley MJ, Tillman SM, Gibbs JD, Elliott MA, Frimel TN, Gibbs CP, Vandenborne K. Muscle adaptations with immobilization and rehabilitation after ankle fracture. Med Sci Sports Exerc. 2004;36:1695-701. https://doi.org/10.1249/01.mss.0000142407.25188.05.

41. Lin C-WC, Donkers NAJ, Refshauge KM, Beckenkamp PR, Khera K, Moseley AM. Rehabilitation for ankle fractures in adults. Cochrane Database Syst Rev. 2012;11:CD005595. https://doi.org/10.1002/14651858.CD005595.pub3.

42. Larsen P, Nielsen HB, Lund C, Sørensen DS, Larsen BT, Matthews M, Vicenzino B, Elsoe R. A novel tool for measuring ankle dorsiflexion: A study of its reliability in patients following ankle fractures, Foot Ankle Surg. Off J Eur Soc Foot Ankle Surg. 2016;22:274-7. https://doi.org/10.1016/j.fas.2016.01.008.

43. Nilsson GM, Jonsson K, Ekdahl CS, Eneroth M. Effects of a training program after surgically treated ankle fracture: a prospective randomised controlled trial. BMC Musculoskelet Disord. 2009;10:118. https://doi.org/10.1186/1471-2474-10-118.

44. Hirschmüller A, Konstantinidis L, Baur H, Müller S, Mehlhorn A, Kontermann J, Grosse U, Südkamp NP, Helwig P. Do changes in dynamic plantar pressure distribution, strength capacity and postural control after intra-articular calcaneal fracture correlate with clinical and radiological outcome? Injury. 2011;42:1135-43. https://doi.org/10.1016/j.injury.2010.09.040.

45. Horisberger $M$, Hintermann B, Valderrabano V. Alterations of plantar pressure distribution in posttraumatic end-stage ankle osteoarthritis. Clin Biomech. 2009;24:303-7. https://doi.org/10.1016/j.clinbiomech.2008.12.005.

46. Jansen H, Fenwick A, Doht S, Frey S, Meffert R. Clinical outcome and changes in gait pattern after pilon fractures. Int Orthop. 2013;37:51-8. https://doi.org/10.1007/s00264-012-1716-1.

47. D’Addio G, Donisi L, Pagano G, Improta G, Biancardi A, Cesarelli M, Agreement between Opal and GWalk Wearable Inertial Systems in Gait Analysis on Normal and Pathological Subjects, in: 2019 41st Annu. Int. Conf. IEEE Eng. Med. Biol. Soc. EMBC, IEEE, Berlin, Germany, 2019: pp. 3286-3289. https://doi.org/10.1109/EMBC.2019.8857841.

48. Donisi L, Pagano G, Cesarelli G, Coccia A, Amitrano F, D’Addio G. Benchmarking between two wearable inertial systems for gait analysis based on a different sensor placement using several 
statistical approaches. Measurement. 2021;173:108642.

https://doi.org/10.1016/j.measurement.2020.108642.

\section{Figures}
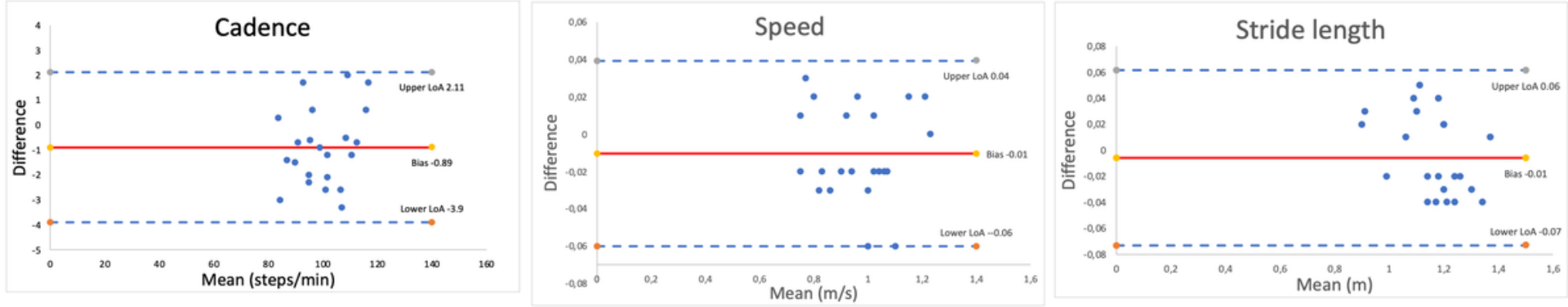

\section{Figure 1}

Bland-Altman plots for cadence, speed, and stride length. Each graph presents the mean difference (solid line) and 1.96-fold standard deviation of difference (dashed line) indicating the limits of agreement between the measurements 